\title{
Estimate Mass Flow Rate of Dense Phased Powder Solids
}

\author{
Usama Abrar ${ }^{1, *}$, Liu $\mathrm{Shi}^{1}$, Nasif Raza Jaffri ${ }^{1}$, Yi $\mathrm{Kang}^{2}$, Muhammad Nawaz ${ }^{3}$, Zhu Huixian ${ }^{1}$ \\ ${ }^{1}$ School of Control and Computer Engineering, North China Electric Power University, Beijing 102206, China \\ ${ }^{2}$ School of Energy Power and Mechanical Engineering, North China Electric Power University, Beijing 102206, China. \\ ${ }^{3}$ Department of Computer Engineering, Bahria University Islamabad Campus, Islamabad, Pakistan.
}

\begin{abstract}
The complex multiphase gas-solid flow has always been a point of attraction for researchers over the past decade to explore the sensing techniques to sense and measure the mass flow. Weather dilute or dense phase flow, the gas-solid flow measurement generally requires velocity profile and volumetric concentration measurement to find the mass flow rate. The nature of the solids, the environmental factorsspecially moisture adversely affects the sensor readings-specifically when it is non-invasive capacitive sensors. Gas-solid flow finds its applications in power plants, food, chemical, automobile, and pharmaceutical industries. This paper aims to explore the evolution of a novel approach of using load cell in conjunction with capacitive electrodes for calculating the mass flow rate of the solids.
\end{abstract}

\section{Introduction}

Powder flow finds its application from everyday domestic kitchen and laundry activities like sugar, coffee, and detergents to the most significant events of nature like a mudslide, avalanches, and pyroclastic flow. Similarly, industries like mining, chemical process industries, pharmaceuticals, agricultural industries, and furnaces also use powder flow to handle the transport of larger volumes from one place to the other. [1] Pneumatic conveying have earned a remarkable name and fame in many chemical, food, power generation, and other process industries. The efficiency of these industries based on the precise measurement of the mass flow rate of solids. The alarming situation of pollution and quickly curtailing natural resources of power have compelled the thermal power plants to enhance its efficiency to avoid any wastage of pulverized coal. [2], [3]. Based upon advancement in technology and sensing methods several flow measurement techniques are available. Digital imaging, optical sensing, acoustic techniques, radiometric methods, electrostatic sensors, and capacitive sensors are the notable techniques used for the measurement of Gas/Solid particles flow rate measurement. However, the choice is based upon the concentration of solid particles. The optical sensors, digital imaging and acoustic techniques give better results when the concentration of solid particles is low. Although hazardous to health by radiation leakage and require proper shielding, the results of radiometric methods are quite promising in the case of high concentration of solid particles.[4], [5]. If an economical, trustworthy, simple, and robust solution is required, the electrostatic and capacitive sensors are the best choices. Online and offline assessments of flow parameters extensively employ capacitance measurement method. [6]
The mass flow rate of solids through a channel can be calculated by mechanically measuring the mass of solid in a certain defined region of channel and mean velocity of the solids. However, this method has some limitations and require some predefined assumptions. Several techniques like resistive sensors, capacitive sensors, magnetic resonance, and tomography in conjunction with spatial filtering, Doppler's shift, and crosscorrelation are used to calculate the velocity of the solid particles. Specific pros and cons are associated with the capacitive sensing techniques used for the calculation of mass flow rate of solids. Its low cost, robustness noninvasive nature supports its application in the industry while low solids/air mass flux ratio, inhomogeneous solids cross-sectional distribution in the pipe and moisture content adversely affects the results of capacitive sensing. With the added advantage of better performance, high safety, and suitability for small and large vessels, capacitive sensors have a cutting edge of precise quantitative remote sensing from a remote location from other tomographic techniques. Nowadays, capacitive tomography has widespread in the industry.[7] Efforts are being made to improvise the system to attain promising benefits from its analysis in the industry. In the flow measurement of solid particles, the nonhomogeneity of the solid particles is a critical issue.

Furthermore, the capacitive electrodes have a variable sensitivity. The particles closer to the wall of the electrodes are easily detectable and provide a significant change in the capacitance values as compared to the solid particles flowing away from the electrodes. So, the accuracy of the capacitive sensor depends upon the location of the electrodes and the flow path of solid particles.

This paper covers the preliminary investigations of the off-line estimation of the mass flow rate of solid

Corresponding author: uabrar@gmail.com 
particles in a channel using mix blend of capacitive sensors assembly and load cells to cover the limitations of each of them. The issues of moisture content effect, the flow path, and the location of sensors overcome by the load cell. However, the velocity measurement using a capacitive sensor pair help to measure the average mass flow rate of solid in a unit cross-section area of the pipe. The main idea revolves around the data acquisition of load cell on a time scale to quantify the mass of the solid particles in a steady state flow through the channel of known length. The load cell attached at the bottom of the channel. Usually, the load cells are connected to the blow tank or receiving tank to measure the MFR. For large scale conveying system, this approach is not applicable since the load cell designed for higher weight values is less accurate than the load cell designed for lower values. However, if the load cell attached at the base of the channel in such a way that the sliding particles flow over the surface of the load cell. It is assumed to give more accurate results for MFR estimation. The capacitive sensors help to find the average velocity of the sand particles. The flow of the sand particles based upon the assumption that the particles are moving parallel to the axis of the pipe and perpendicular to the plane of the sensor such that every passing particle has to flow through both the capacitive sensors.

\section{Powder flow theory}

The flow pattern of solid particles depends upon the nature of the material. However, several external factors like temperature, the angle of repose and the moisture content also effect the flow behavior. The cohesiveness of particles plays a key role in flow pattern which is raised by moisture content. The temperature variations also change the cohesive strength. Cohesiveness is also dependent upon particle size. Finer powders are more cohesive also they have higher wall friction and thus challenging to flow smoothly. A cohesive bond is formed when solid particles stay at rest for a long time. A chemical reaction, crystallization, or adhesive bonding can also cause this. Sometimes, after a cohesive arch is broken up, say by somehow initiating flow, the material can revert to its original flow condition and not exhibit a similar cohesion if left at rest again. On the other hand, some materials will time and again, bridge and rat-hole even after the flow is re-initiated [8]. Factors primarily affecting the powder flow are of three types. Powder properties; (particle size, shape, density, morphology, size distribution, etc.); processing operating conditions applied by the production equipment; including air-powder interactions and environment (humidity, temperature, storage conditions, etc.) [9].

The mass flow rate of solid particles in a channel is given by equation 1 .

$$
M(t)=\rho_{s} A V_{s}(t) \beta_{s}(t)
$$

$\rho_{s} \quad$ The density of the Solids

A Effective Cross Section Area of the Pipe

$V_{s}(t) \quad$ The velocity of the Particle

\section{$\beta_{s}(t)$ Volumetric Concentration of the Solids}

Solid particles, sliding in an inclined surface move as discrete dense phase based upon the Euler- Lagrangian model. Air being fluid is a continuous medium, while the particle phase is distinct. The particle's movement based upon the instantaneous collision of the particles itself; controlled by the momentum of the particles; and the suspension movement that is governed by the drag force.

\section{Experimental setup}

The main components of an Electric Capacitance Assembly system are the sensor, the data acquisition circuit, and the computer. The sensor is a single or more pair of metallic electrodes whose dimension and mutual distances are adjustable as per requirements. These electrodes are mounted on the insulated pipe, which is extraneously covered with an earthed metallic sheet to reduce the noise impact. The data acquisition circuit excite the capacitive electrode pairs the resultant capacitance value is digitized for data acquisition. The computer stores and analyses the data by applying different mathematical techniques [10]

The block diagram of the experimental setup is shown in figure 1 . The solid particles flow through a rectangular, insulated, and inclined channel- fixed on a stand, and the angle of inclination can be varied as per requirements. The dimensions of the channel are $1 \times 2 \times 33$ $\mathrm{cm}^{3}$. Two pairs of electrodes 'Sensor 1' and 'Sensor 2' of sizes $2 \times 1 \mathrm{~cm}^{2}$ and $1 \times 1 \mathrm{~cm}^{2}$ respectively are fixed on the lower and upper surface of the channel $11.5 \mathrm{~cm}$ (distance between their centers) apart such that all the sand particles flowing through 'sensor 1' must flow through 'sensor 2'. The channel is enclosed in a metallic shielding attached to a common ground with protective metallic shielding attached at the upper and lower ends of both the sensors to minimize the effects of the noise signals. A load cell is connected to the channel. It calculates the mass of solid particles present on the entire length of the channel.

The data from the load cell using IC $\mathrm{Hx} 711$ to interface with Arduino UNO and from metallic sensors using Eval-AD7746EBZ and Arduino UNO is received and stored in a computer using time synchronization. Eval-AD7746EBZ is a capacitance to digital converter evaluation board which is primarily used as a data acquisition circuit for the measurement of capacitance between the electrodes. The primary significance of AD7746EBZ is its high resolution of 24-bits down up to $4 \mathrm{aF}$ and accuracy up to $4 \mathrm{fF}$. Its full-scale capacitance rage is $\pm 4 \mathrm{pF}$. The maximum sampling rate of the device is $90 \mathrm{~Hz}$. The capacitance to be measured is directly connected to its terminals- Excitation terminal and Input terminal. It communicates through the $\mathrm{I} 2 \mathrm{C}$ interface with microcontroller-Arduino-UNO. The registers were configured according to the Data Sheet for single mode capacitance measurement using I2C communication protocol. [11] 


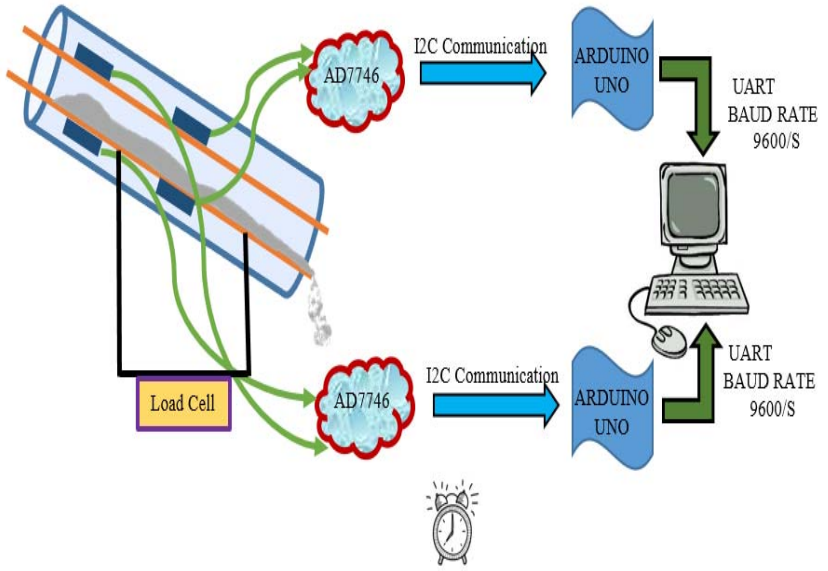

Fig. 1. Block Diagram of the Experimental Setup

The raw data from Eval-AD7746EBZ is received by Arduino and sent to the Computer through UART where the values were fed in the formula to calculate the capacitance of both the sensors. Concurrently the data from the load cell using $\mathrm{Hx} 711$ interface and Arduino $\mathrm{UNO}$ is also received at the computer. The computer program also gives the preferred sampling interval to the Microcontrollers that starts dropping off and thus stores the data of electrode sensors and load cell at the required sampling rate.

\subsection{Velocity measurement}

Cross-correlation is used to analyze the velocity of the solid particles flowing through a channel as shown in figure 2 . The cross-correlation of the two signals $\mathrm{x}(\mathrm{t})$ and $\mathrm{y}(\mathrm{t})$ is defined by equation 2

$$
C(\tau)=\frac{1}{\tau} \int_{0}^{\tau} x(t) y(t+\tau) d \tau
$$

Where $T \rightarrow \infty$

The above equation shows the resemblance between $\mathrm{x}(\mathrm{t})$ and $\mathrm{y}(\mathrm{t})$ while shifting $\mathrm{y}(\mathrm{t})$ along the time axis to $y(t+\tau)$. For a specific value of $\tau=\tau_{\max }$ where $C(\tau)$ gives the maximum value, both $\mathrm{x}(\mathrm{t})$ and shifted $\mathrm{y}(\mathrm{t})$ is said to be most alike. If $x(t)$ is the signal from the 'Sensor 1' and $y(t)$ is the signal from the 'Sensor 2'. Since both the sensors are attached along the axis of the pipe in such a way that the solid particles flowing from the first sensor after a delay passes through the second sensor, the signals from both the sensors are the same with a difference of time delay which is the time required by the solid particles to flow from first sensor to the second sensor. For a known distance between the sensors, the average velocity of the particles can be found by taking the fraction between the distance $\mathrm{D}$ and the time $\tau_{\max }$. In real time scenario, the data stored from both the sensors as discrete time values, so the modified cross-correlation of discrete time signals being unable to extend $\mathrm{T} \rightarrow \infty$ is defined by equation 3

$$
C[m]=\frac{1}{2} \sum_{n=0}^{L} x[n] y[n+m]
$$

Where $\mathrm{L}$ is the length of the frame size, used to calculate the cross-correlation. It is the number of samples in a summation. $\mathrm{m}$ is the number of delayed samples-time delay. In other words, $\mathrm{L}$ number of samples of $x[n]$ is multiplied by $L$ number of samples of shifted $y[n+m]$. If $\Delta t$ is the sampling interval, the time to obtain the peak of the cross correlation function given by $\tau_{\max }=\mathrm{m} * \Delta \mathrm{t}$. The average velocity of the solid particles is given by equation 4

$$
V=D / \tau_{\max }
$$
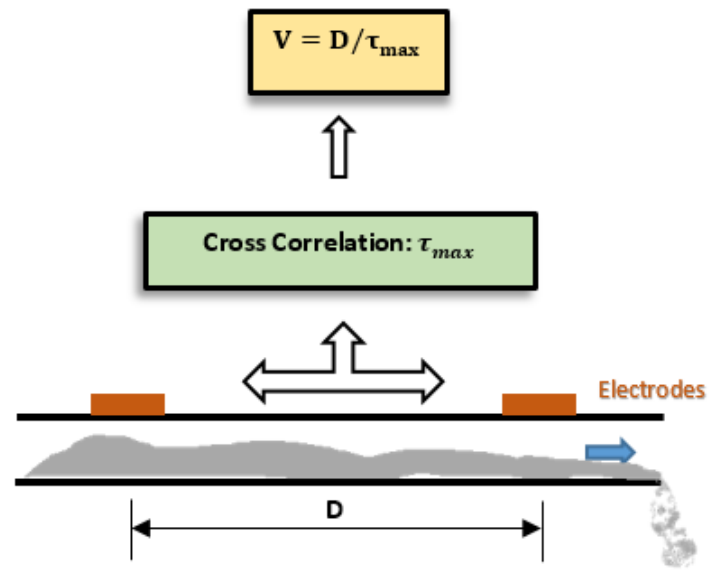

Fig. 2. Velocity of Solid Particles Using Cross-Correlation

The choice of a suitable distance between the two sensors is essential. If the sensors placed at a farther distance, the slow data acquisition rate may occur.[12] However, the better similarity between the signals is possible if the gap between the sensors is small. Although there is a chance of cross-interference of the signals of the two sensors if they are very close to each other. So, the distance between the sensors is chosen depending upon the similarity of the signals and the dynamic behavior of the system. [2], [10]

\subsection{Mass flow rate measurement}

Velocity measurement gives the average value of the velocity of solid particles flowing in a channel. However, the actual flow velocity is variable being lowest at the base surface and highest in the center where the gassolid phases interact with each other. If $\mathrm{V}$ is the velocity value obtained from cross-correlation, and the value of load cell attached at the base of the known length channel ${ }^{l}$-observing a steady flow is $m$. The equation 5 can compute the average mass flow rate $M F R$ at a crosssection of the pipe

$$
M F R=\frac{m}{l} * V
$$

The equation is valid only for the steady state flow of the solid particles sliding along the bottom surface of the channel as a discrete dense phase material under gravity. If the particles pneumatically conveyed in air, they dispersed, and the chances of errors in load cell measurement are possible. Also, dilute phase suspended solid particle do not produce a significant effect in capacitive readings. 


\section{Experimental results}

\subsection{Velocity measurement}

Table 1 shows the average velocity of solid particles for several iterations through an inclined channel fixed at an angle of $50^{\circ}$. Figure 3 shows the capacitance values of sensor 1 and Sensor 2 as the solid particles are allowed to flow through the channel. The sampling interval of 10 $\mathrm{ms}$ is adjusted for the record of data. The similarity of variation in the pattern of both signals indicates the flow of solid particles through both the sensors. The separation between the pair of the electrodes of the individual sensor is approximately $1 \mathrm{~cm}$ (outer diameter of the pipe) Dried sand particles are allowed to flow through the pipe slowly. The sand particles behave as dynamically flowing dielectric medium between the electrodes of the sensors. The nature of the material $\left(\mathrm{SiO}_{2}\right)$ and its concentration comparative to the diameter of the channel (space between the electrodes) is believed to give a proper signal to noise ratio. For the empty channel, the average value of 'Sensor 1' and 'Sensor 2' are $0.0052 \mathrm{pF}$ and $0.0046 \mathrm{pF}$, respectively. The slight variations in both the signals are because of the noise that can be reduced by subtracting the average value of the signal from individual stored sample of the data, as shown in figure 4.

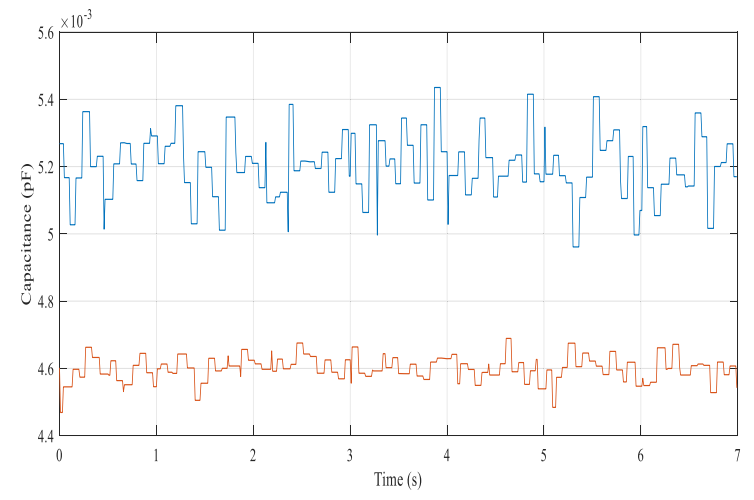

Fig. 3. Output Signals of Capacitive Sensors when the Distance is $11.5 \mathrm{~cm}$

The amplitude of both the signal is different since the size of the electrode pairs of both the sensors is changed. However, the pattern of variations repeats itself after an interval that indicates the steady flow of sand in the channel.

Table 1. Velocity Of Solid Particles Using Cross-Correlation

\begin{tabular}{|l|l|l|l|}
\hline $\begin{array}{l}\text { Sr. } \\
\text { No. }\end{array}$ & $\begin{array}{l}\text { Time } \\
\text { Estimated }(\mathrm{s})\end{array}$ & $\begin{array}{l}\text { Distance } \\
\text { Between the } \\
\text { Sensors }(\mathrm{cm})\end{array}$ & $\begin{array}{l}\text { Velocity } \\
\text { Estimated } \\
(\mathrm{m} / \mathrm{s})\end{array}$ \\
\hline 1 & 0.29 & 11.5 & 0.3966 \\
\hline 2 & 0.24 & 11.5 & 0.4792 \\
\hline 3 & 0.18 & 11.5 & 0.6389 \\
\hline
\end{tabular}

The cross-correlation result is shown in figure 5 . The maximum peak is obtained at the $24^{\text {th }}$ discrete interval. Since the data is sampled at $10 \mathrm{~ms}$ sampling interval, the average time required by the solid particles to flow from
'Sensor 1' to 'Sensor 2' is $240 \mathrm{~ms}$. Since the distance between the centers of both the sensors is $11.5 \mathrm{~cm}$. So the average velocity of the solid particles is $0.479 \mathrm{~m} / \mathrm{s}$ using equation 4

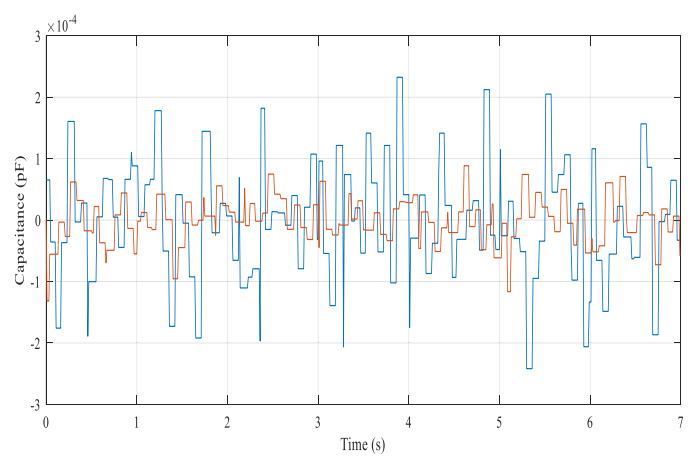

Fig. 4. Output Signals of both the Sensors after Noise Reduction

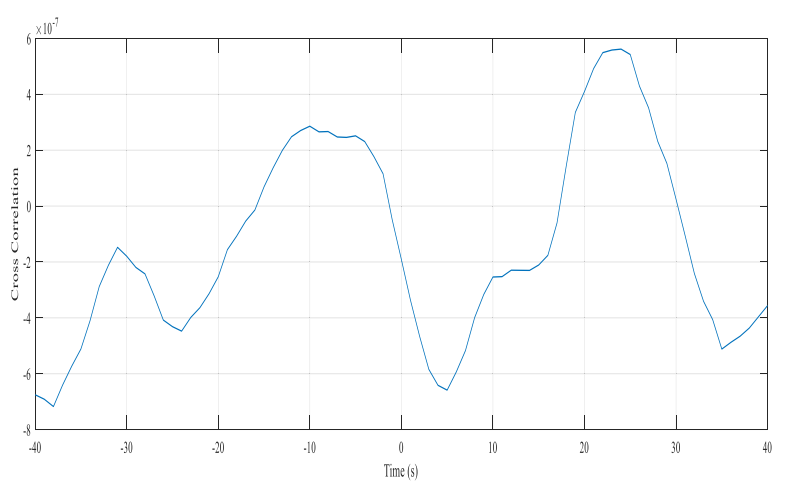

Fig. 5. Cross-Correlation between the two Sensors when the Distance Between them is $11.5 \mathrm{~cm}$

\subsection{Mass flow measurement}

As evident from figure 1, the load cell readings after calibration were also recorded with time synchronization at the same time interval as that of 'Sensor 1' and 'Sensor 2'.From the values of table 1 and using equation 5 , the mass flow rate of the solid particles through the channel is shown in figure 6 .

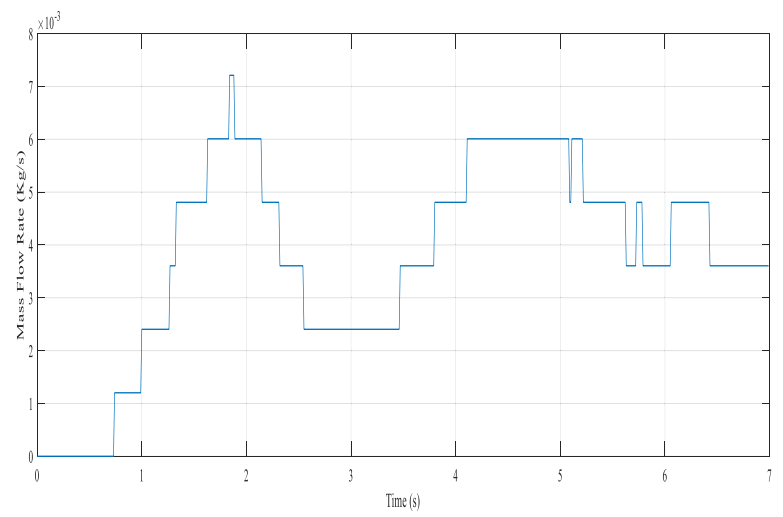

Fig. 6. Mass Flow Rate of Solid Particles in an Inclined Channel

The above graph indicates the instantaneous values of the mass flow rate through a channel. It is essential to mention here that the inlet flow concentration of solid 
particles was not possible to keep constant using the manual insertion of the particles in the channel. As a result, the mass flow rate kept changing. The max value of mass flow rate attained was $7.2 \times 10^{-3} \mathrm{~kg} / \mathrm{s}$, as shown in figure 6.

\section{Conclusion and future work}

This paper focuses on the preliminary investigations on the calculation of the average mass flow rate of solid particles in an inclined channel using a mechanical sensor. The results thus obtained are fluctuating because the flow of the solid particles was kept variable. It is assumed that the flow was consistent throughout the channel. However, the average value of the flow can be closer to the actual value if the length of the channel under observation is kept as small as possible. However, there are some problems in the above studies, the volumetric concentration of solid particles at the inlet in the experimental setup was unknown. In the future, the impact of different velocities of air will be observed on the motion of the solid particles. Similarly, the movement of particles of different diameters under the same air velocity profile will be followed. Also, a fusion of electrical and mechanical sensor outputs will be used for better measurement of the mass flow rate of solid particles in an inclined channel.

Acknowledgment

The authors wish to extend their gratitude to NSFC for sponsoring the research (No 61571189, 61871181) and to the State Administration of Foreign Experts Affairs for supporting the 111 projects (ref: B13009).

\section{References}

1. T. Dias, W. Melchert, M. Kamogawa, F. Rocha, and E. Zagatto, "Fluidized particles in flow analysis: potentialities, limitations and applications," Talanta, vol. 184, , pp. 325-331, 2017.

2. H. Seraj, M. Rahmat, and M. Khalid, "Measurement of velocity of solid/air two phase fluid using electrostatic sensors and cross correlation technique," Sci. Iran., vol. 20, no. 3, pp. 786-792, 2013.
3. S. Rao, K. Zhu, C. Wang, and S. Sundaresan, "Electrical capacitance tomography measurements on the pneumatic conveying of solids," Ind. Eng. Chem. Res., vol. 40, no. 20, pp. 4216-4226, 2001.

4. C. Hartmann, J. Wang, D. Opristescu, and W. Volk, "Implementation and evaluation of optical flow methods for two-dimensional deformation measurement in comparison to digital image correlation," Opt. Lasers Eng., vol. 107, no. September 2017, pp. 127-141, 2018.

5. M. Lima, S. Andrade, I. Barreto, L. Almeida, and M. Araújo, "A digital image-based micro-flow-batch analyzer," Microchem. J., vol. 106, pp. 238-243, 2013.

6. W. Zhang, C. Wang, W. Yang, and C. H. Wang, "Application of electrical capacitance tomography in particulate process measurement - A review," Adv. Powder Technol., vol. 25, no. 1, pp. 174-188, 2014.

7. H. Che, M. Wu, J. Ye, W. Yang, and H. Wang, "Monitoring a lab-scale wurster type fluidized bed process by electrical capacitance tomography," Flow Meas. Instrum., vol. 62, no. September 2017, pp. 223-234, 2018.

8. H. Lu, X. Guo, Y. Jin, and X. Gong, "Effect of moisture on flowability of pulverized coal," Chem. Eng. Res. Des., vol. 133, pp. 326-334, 2018.

9. W. Chen and A. Roberts, "A modified flowability classification model for moist and cohesive bulk solids," Powder Technol., vol. 325, pp. 639-650, 2018.

10. M. Sun, S. Liu, J. Lei, and Z. Li, "Mass flow measurement of pneumatically conveyed solids using electrical capacitance tomography," Meas. Sci. Technol., vol. 19, no. 4, 2008.

11. Z. Huang, J. Zhu, and L. Lu, "An AD7746-Based Data Acquisition System for Capacitive Pressure Sensor in Weather Detection Application," Key Eng. Mater., vol. 483, pp. 461-464, Jun. 2011.

12. K. Grudzień, Z. Chaniecki, and L. Babout, "Study of granular flow in silo based on electrical capacitance tomography and optical imaging," Flow Meas. Instrum., vol. 62, no. September 2017, pp. 186-195, 2018 